\title{
PROPIEDADES GEOMÉTRICAS DE LOS BLOQUES Y LA MAMPOSTERÍA DE CONCRETO
}

\author{
Alejandro Navas Carro \\ Julio César Salazar Méndez.
}

\begin{abstract}
Resumen
En este documento, se analizan las propiedades geométricas del bloque y la mampostería de concreto en el país. Estas propiedades son: dimensiones y espesores de pared del bloque de concreto; también, el área, inercia y módulo de sección transversales netos, además del espesor equivalente de la mampostería de concreto.

La etapa experimental contempló el muestreo de 270 bloques de concreto en 16 de las 20 bloqueras de mayor producción en el país, las cuales se encuentran distribuidas a lo largo y ancho del territorio nacional. Estas muestras se utilizaron para el ensayo de área neta en el bloque de concreto, basado en la norma ASTM C140. Con los resultados de este ensayo, se obtuvieron las propiedades geométricas con base en un análisis estadístico de los datos, empleando principalmente los percentiles de la muestra.

Los resultados obtenidos muestran espesores de pared mayores a los mínimos establecidos por la norma INTE, por lo que se recomienda emplear en el diseño, un espesor de pared de $24 \mathrm{~mm}, 26 \mathrm{~mm}$ y $28 \mathrm{~mm}$ para los bloques de $12 \mathrm{~cm}, 15 \mathrm{~cm}$ y $20 \mathrm{~cm}$ de espesor respectivamente, además de un espesor de pared de $25 \mathrm{~mm}$ para el bloque modular.
\end{abstract}

Palabras clave: bloques de concreto; ensayo de área neta; propiedades geométricas; mampostería de concreto.

\begin{abstract}
In this document, the geometrical properties of block and concrete masonry in the country have been analyzed. These properties are: dimensions and wall thickness of concrete masonry units, also the net cross sectional area, inertia and section modulus, in addition to the equivalent thickness of concrete masonry walls.

The experimental phase contemplated 270 concrete masonry units in 16 of the 20 more productive factories in Costa Rica, which are distributed throughout the country. These samples were used for net area test in the concrete masonry units based on ASTM C140. With these results, the geometrical properties of the masonry units were obtained based on a statistical analysis of the data using mainly the percentiles of the sample.

The results show wall thicknesses greater than the minimum specified by the INTE norm, reason why it is recommended to use a wall thickness of $24 \mathrm{~mm}, 26 \mathrm{~mm}$ and $28 \mathrm{~mm}$ for masonry units of $12 \mathrm{~cm}, 15 \mathrm{~cm}$ and $20 \mathrm{~cm}$ in thickness respectively, and a wall thickness of $25 \mathrm{~mm}$ for the modular block.
\end{abstract}

Keywords: concrete blocks; net area test; geometrical properties; concrete masonry.

Recibido: 20 de mayo de 2014 • Aprobado: 8 de julio de 2014

\section{INTRODUCCIÓN}

El International Building Code [IBC] (2003) señala lo siguiente:

La palabra "mampostería" es un término general que se refiere a la construcción utilizando unidades prefabricadas de arcilla, hormigón, baldosas de arcilla, bloques de vidrio, piedras naturales y similares. Uno o más tipos de unidades de mampostería se unen entre sí con mortero, piezas de metal, refuerzo y accesorios para formar paredes y otros elementos estructurales. (p. 21-1).

En Costa Rica, se cuenta con diversos tipos de mampostería hueca que principalmente se dividen en bloques modulares y bloques de $12 \mathrm{~cm}, 15 \mathrm{~cm}$ 
y $20 \mathrm{~cm}$ de espesor. Para efectos de este artículo, se denomina viga bloque al bloque de concreto que permite que el refuerzo horizontal se embeba completamente en concreto. La Figura 1 ilustra bloques convencionales, ranurados y viga bloque.

La mampostería se utiliza con propósitos estructurales y no estructurales; estructurales para la construcción de edificaciones, muros de retención, columnas, vigas, fundaciones, tapias, entre otros; mientras que no estructural para divisiones y usos decorativos. En esta materia, el Código Sísmico de Costa Rica 2010 define en su Apéndice A los requisitos complementarios para mampostería estructural.

En materia legal, las normativas para el control de calidad de los bloques y los requisitos que deben cumplir, han evolucionado a lo largo del tiempo desde que se publicaran las primeras normativas. La primera de ellas se publicó en el primer Código Sísmico de Costa Rica en 1974 y la segunda fue el primer reglamento nacional, publicado por el Ministerio de Economía, Industria y Comercio (MEIC) en 1976.

En la actualidad, el Instituto de Normas Técnicas de Costa Rica (INTECO) es el encargado de la homologación de las normas según la Ley de la Calidad, de manera que el control de calidad de los bloques de concreto se establece en algunas de las normas INTE, las cuales son de uso voluntario. En estas normas, se definen los métodos de ensayo y muestreo, los requisitos de la mampostería hueca para uso estructural y no estructural, la calidad de los agregados, concreto de relleno y mortero de pega de bloques, entre otros requisitos. Las normas INTE se basan en normas internacionales como las ASTM. Cabe destacar que a diferencia de las normas INTE, el CSCR es de acatamiento obligatorio.

Desde la época de los años noventa, se han venido realizan investigaciones de manera intensa con respecto al comportamiento y propiedades mecánicas de bloques y elementos de mampostería; sin embargo, no se han realizado estudios para determinar las dimensiones de estos elementos.

Las propiedades geométricas de la mampostería de concreto son de importancia para la determinación de área neta, densidad y cálculos de capacidad a cortante y flexión, así como la determinación de la rigidez. Por ende, son indispensables para el diseño de estructuras de mampostería que cumplan con los requerimientos descritos en el Código Sísmico de Costa Rica 2010 y que cuenten con un comportamiento estructural adecuado.

Este es el primer estudio realizado en el país para determinar las propiedades geométricas de los elementos de mampostería. Para ello, se utilizaron muestras de las principales fábricas de bloques a nivel nacional, ubicadas en distintas partes del país. Tiene como objetivos principales informar acerca de la distribución nacional de las empresas fabricantes de mampostería y evaluar las características geométricas de bloques de concreto y mampostería elaborados en Costa Rica, específicamente: dimensiones y espesores de pared de los bloques; área, inercia y módulo de sección transversales netos; y espesor equivalente de la mampostería de concreto. El presente estudio no tiene como propósito la investigación ni divulgación de la calidad de estos elementos.

Los resultados son de importancia pues permiten determinar los rangos de variación de las dimensiones reales de los bloques de mampostería fabricados y compararlos con la normativa vigente, así como determinar las propiedades geométricas reales de la mampostería de concreto. Esto con miras a brindar información esencial para los diseñadores y constructores de sistemas a base de mampostería, pues puede permitir elaborar diseños y obras con un mayor grado de seguridad y eficiencia.

Cabe destacar que este muestreo se realizó entre los meses de octubre del año 2012 y abril del 2013 y por ende, las conclusiones obtenidas a partir de este estudio son válidas únicamente para este espacio de tiempo en específico. Esto debido a que el procedimiento de fabricación de los bloques puede experimentar variaciones en el tiempo.

\section{PRODUCTORES DE BLOQUES DE CONCRETO EN COSTA RICA}

El mercado de bloques de concreto en Costa Rica ha sufrido diversos cambios en los últimos años. Actualmente existen aproximadamente 20 bloqueras reconocidas, las cuales producen bloques de diversos tamaños, desde los más comunes de $12 \mathrm{~cm}, 15 \mathrm{~cm}$ y $20 \mathrm{~cm}$ de espesor, viga bloque y bloque modular, hasta bloques 


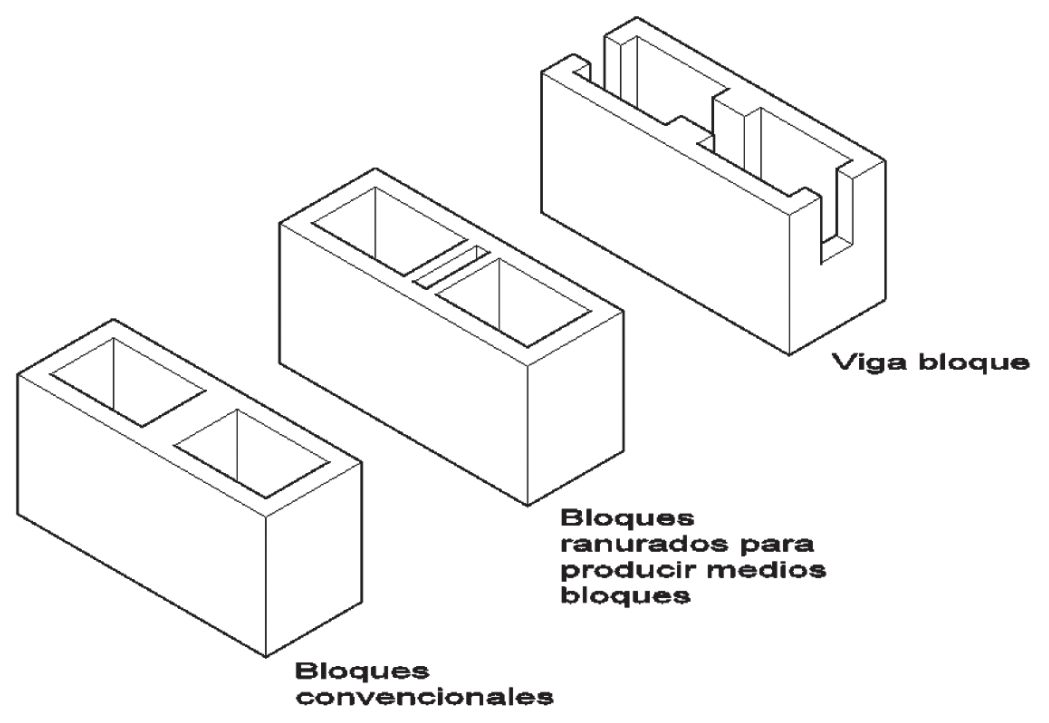

Figura 1. Bloques de concreto más comunes.

Tabla 1. Bloqueras de concreto de mayor producción en Costa Rica.

\begin{tabular}{ll}
\hline Nombre & $\begin{array}{l}\text { Ubicación de la } \\
\text { planta }\end{array}$ \\
\hline Quebadores del Sur & Pérez Zeledón \\
Concreto Industrial & Guápiles \\
Concreto Industrial & Santa Ana \\
Pedregal & Belén \\
Pedregal & Nicoya \\
Pedregal & Barranca \\
Pedregal & San Carlos \\
Productos de Concreto & Guápiles \\
Productos de Concreto & Ochomogo \\
Productos de Concreto & San Rafael de \\
Productos de Concreto & Chajuela \\
Bloquera el Progreso & Alajuela \\
Concrepal & Palmares \\
Concrepal & Barranca \\
Concrepal & Liberia \\
Concrepal & Parrita \\
Bloquera Irazú & Coronado \\
Bloquisa & Desamparados \\
Bloquera la Pista & Grecia \\
Concreprefa & Guápiles \\
\hline & \\
\hline &
\end{tabular}




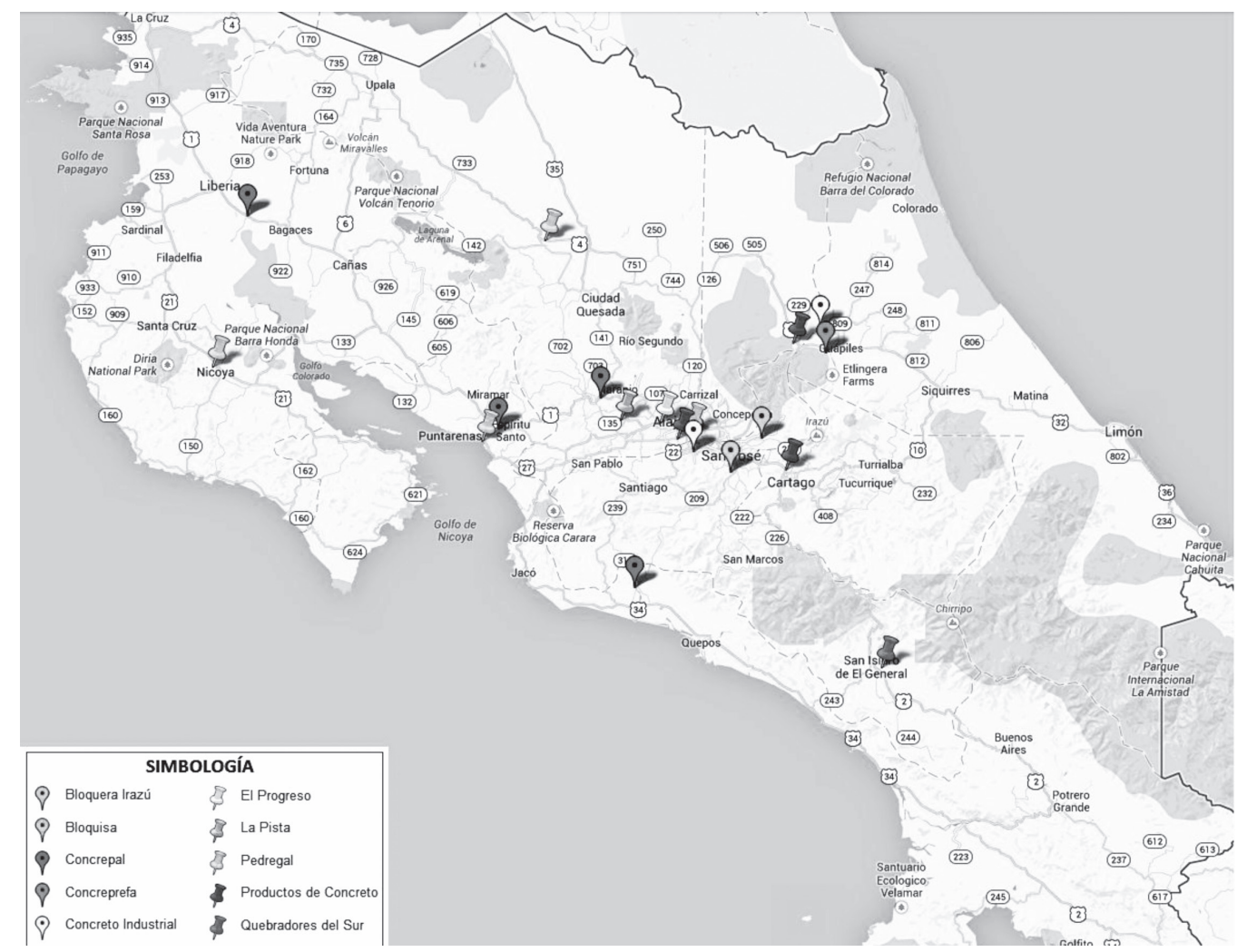

Figura 2. Ubicación de las plantas de las bloqueras de mayor producción en Costa Rica.

Nota: Salazar, J. (2013). Propiedades Geométricas y Densidad de Mampostería de Concreto. Proyecto de graduación para optar por el grado de Licenciatura en Ingeniería Civil, Escuela de Ingeniería Civil, Universidad de Costa Rica , Costa Rica.

específicos de algunas empresas como el Masterblock y el Superbloque.

De estas 20 bloqueras, se pueden encontrar 10 empresas reconocidas y diferentes en el mercado nacional, las cuales cuentan con plantas en todas las provincias del país. Una lista detallada de las empresas que fabrican bloques, sus plantas y su ubicación, se presenta en la Tabla 1.

Como se observa en la Tabla 1, la mayoría de las bloqueras se encuentran en las provincias de San José y Alajuela. La zona del Caribe cuenta con muy pocas bloqueras y en la provincia de Limón, específicamente, las bloqueras más cercanas se encuentran en Guápiles y Chirripó. Asimismo en la zona de Heredia, Cartago, Guanacaste y Puntarenas, se encuentran una, una, dos y tres bloqueras respectivamente.

En la Figura 2, se muestra un mapa con la distribución de las bloqueras de mayor producción en Costa Rica.

Todas las empresas fabrican bloques de $12 \mathrm{~cm}, 15 \mathrm{~cm} \mathrm{y} 20 \mathrm{~cm}$ de ancho; mientras que únicamente 4 empresas fabrican bloques modulares y solo en algunas de sus plantas. En la Tabla 2 se muestra el detalle de los bloques que se fabrican en cada bloquera. 
Tabla 2. Bloques fabricados en las plantas de las bloqueras de mayor producción en Costa Rica.

\begin{tabular}{|c|c|c|c|c|c|}
\hline \multirow{2}{*}{ Nombre } & \multirow{2}{*}{$\begin{array}{c}\text { Ubicación de la } \\
\text { planta }\end{array}$} & \multicolumn{4}{|c|}{ Tamaño de bloque según ancho } \\
\hline & & $12 \mathrm{~cm}$ & $15 \mathrm{~cm}$ & $20 \mathrm{~cm}$ & Modular \\
\hline Quebadores del Sur & Pérez Zeledón & $\sqrt{ }$ & $\sqrt{ }$ & $\sqrt{ }$ & $\mathrm{x}$ \\
\hline Concreto Industrial & Guápiles & $\sqrt{ }$ & $\sqrt{ }$ & $\sqrt{ }$ & $\sqrt{ }$ \\
\hline Concreto Industrial & Santa Ana & $\sqrt{ }$ & $\sqrt{ }$ & $\sqrt{ }$ & $\sqrt{ }$ \\
\hline Pedragal & Belén & $\sqrt{ }$ & $\sqrt{ }$ & $\sqrt{ }$ & $\sqrt{ }$ \\
\hline Pedragal & Nicoya & $\sqrt{ }$ & $\sqrt{ }$ & $\sqrt{ }$ & $\sqrt{ }$ \\
\hline Pedragal & Barranca & $\sqrt{ }$ & $\sqrt{ }$ & $\sqrt{ }$ & $\mathrm{x}$ \\
\hline Pedragal & San Carlos & $\sqrt{ }$ & $\sqrt{ }$ & $\sqrt{ }$ & $\mathrm{x}$ \\
\hline Productos de Concreto & Guápiles & $\sqrt{ }$ & $\sqrt{ }$ & $\sqrt{ }$ & $\sqrt{ }$ \\
\hline Productos de Concreto & Ochimogo & $\sqrt{ }$ & $\sqrt{ }$ & $\sqrt{ }$ & $\mathrm{x}$ \\
\hline Productos de Concreto & $\begin{array}{l}\text { San Rafael de } \\
\text { Alajuela }\end{array}$ & $\sqrt{ }$ & $\sqrt{ }$ & $\sqrt{ }$ & $\mathrm{x}$ \\
\hline Productos de Concreto & Chirripó & $\sqrt{ }$ & $\sqrt{ }$ & $\sqrt{ }$ & $\mathrm{x}$ \\
\hline Bloquera el Progreso & Alajuela & $\sqrt{ }$ & $\sqrt{ }$ & $\sqrt{ }$ & $\mathrm{x}$ \\
\hline Concrepal & Palmares & $\sqrt{ }$ & $\sqrt{ }$ & $\sqrt{ }$ & $\mathrm{x}$ \\
\hline Concrepal & Barranca & $\sqrt{ }$ & $\sqrt{ }$ & $\sqrt{ }$ & $\mathrm{x}$ \\
\hline Concrepal & Liberia & $\sqrt{ }$ & $\sqrt{ }$ & $\sqrt{ }$ & $\sqrt{ }$ \\
\hline Concrepal & Parrita & $\sqrt{ }$ & $\sqrt{ }$ & $\sqrt{ }$ & $\mathrm{x}$ \\
\hline Bloquera Irazú & Coronado & $\sqrt{ }$ & $\sqrt{ }$ & $\sqrt{ }$ & $\mathrm{x}$ \\
\hline Bloquisa & Desamparados & $\sqrt{ }$ & $\sqrt{ }$ & $\sqrt{ }$ & $\mathrm{x}$ \\
\hline Bloquera la Pista & Grecia & $\sqrt{ }$ & $\sqrt{ }$ & $\sqrt{ }$ & $x$ \\
\hline Concreprefa & Guápiles & $\sqrt{ }$ & $\sqrt{ }$ & $\sqrt{ }$ & $\mathrm{x}$ \\
\hline
\end{tabular}

\section{PROGRAMA EXPERIMENTAL}

\subsection{Parámetros técnicos y metodología}

Los ensayos experimentales se llevaron a cabo en las instalaciones del Laboratorio Nacional de Materiales y Modelos Estructurales de la Universidad de Costa Rica (LanammeUCR).

Como se mencionó anteriormente, las normas INTE establecen diversos parámetros para la mampostería de concreto en el país, entre ellos, requisitos geométricos de las unidades de mampostería según el tamaño del bloque, como se muestra en el Tabla 3.
La norma INTE establece que ninguna dimensión (espesor, longitud, altura) podrá diferir por más de $3 \mathrm{~mm}$ de las dimensiones especificadas. Para el cálculo del área neta del bloque de concreto, se siguió el procedimiento de la norma INTE 06-02-13: Muestreo y ensayo de unidades de mampostería de concreto (bloques de concreto), la cual se basa en la norma ASTM C140. Por otro lado, el área bruta del bloque de concreto se determinó como el producto del largo por el ancho.

Como el propósito de este estudio es determinar las dimensiones típicas de las unidades de mampostería de concreto que se producen a nivel nacional y no simplemente 
Tabla 3. Espesores mínimos de las paredes internas de los bloques.

\begin{tabular}{cc}
\hline $\begin{array}{c}\text { Ancho nominal del } \\
\text { bloque (mm) }\end{array}$ & $\begin{array}{c}\text { Espesor mínimo de las } \\
\text { paredes Promedio de tres } \\
\text { unidades (mm) }\end{array}$ \\
\hline 120 & 19 \\
150 & 25 \\
200 & 32 externas y 25 internas \\
\hline
\end{tabular}

Nota: INTE 06-03-01: Elementos de mampostería hueca de concreto (bloques de concreto) para uso estructural Requisitos.

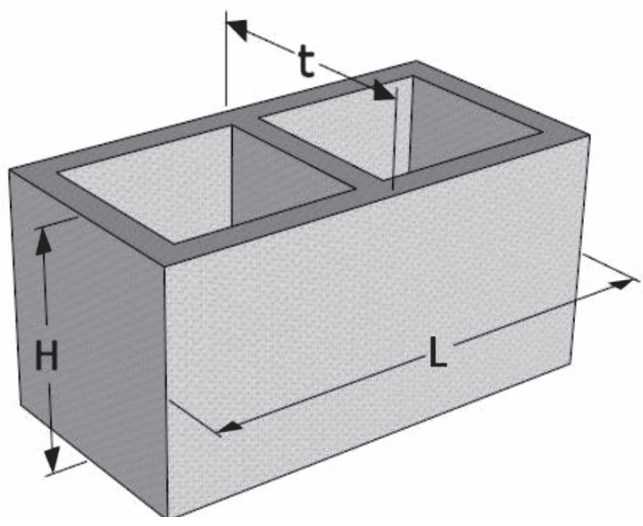

Figura 3. Medición de la altura, espesor y largo del bloque.

una verificación de los mínimos que establece la norma INTE 06-03-01, las mediciones de los bloques se realizaron de la siguiente manera, mediante el uso de vernier:

1. Altura (H): se mide al centro de las 4 caras (4 mediciones), ver Figura 3.

2. Espesor (t): se mide al centro de las caras huecas (2 mediciones), ver Figura 3.

3. Largo (L): se mide al centro de las 2 caras correspondientes ( 2 mediciones), ver Figura 3.

4. Espesores de pared: se miden según la cantidad de celdas del bloque. Si el bloque cuenta con 1 celda, 2 celdas o 3 celdas, se miden 4, 5 o 6 espesores respectivamente. Además, se miden los espesores en ambas caras de falla o caras huecas del bloque, ver Figura 4.
Con respecto a los espesores de pared de los bloques $\left(\mathrm{t}_{\mathrm{f}}\right)$, se debe destacar que por su proceso de fabricación, el bloque es más angosto en una de sus caras de falla, o caras huecas, para que el molde sea fácil de retirar una vez que se da la consolidación, lo cual genera que los espesores de pared en una cara del bloque sean mayores que los espesores de pared en la cara opuesta. Aunque la norma indica que las mediciones se deben tomar en la cara de menor espesor, para este estudio se midieron los espesores de pared en ambas caras de falla del bloque, ver Figura 5.

\subsection{Características de la muestra}

La muestra utilizada para determinar las propiedades geométricas fue de 270 bloques de concreto provenientes de las 16 bloqueras incluidas en el estudio, de manera que se muestrearon 5 bloques de cada tipo en cada una de las bloqueras, los cuales se distribuyen de la siguiente manera.

- 80 bloques de $12 \mathrm{~cm}$ de espesor

- 5 bloques modulares de $15 \mathrm{~cm}$ de longitud nominal

- 75 bloques de $15 \mathrm{~cm}$ de espesor

- 15 bloques modulares de $30 \mathrm{~cm}$ de longitud nominal

- 80 bloques de $20 \mathrm{~cm}$ de espesor

- 15 bloques modulares de $45 \mathrm{~cm}$ de longitud nominal

La norma ASTM C140 indica que se deben utilizar tres muestras de cada tipo de bloque para cada bloquera; sin embargo, se decidió utilizar un número superior de muestras con el fin de obtener resultados más representativos. En el caso del bloque modular, todos los bloques muestreados son similares en cuanto a dimensiones, al bloque modular conocido como Tecnoblock, el cual fue desarrollado por el Instituto Tecnológico de Costa Rica (ITCR); de manera que en este artículo, todos los resultados obtenidos para los bloques 

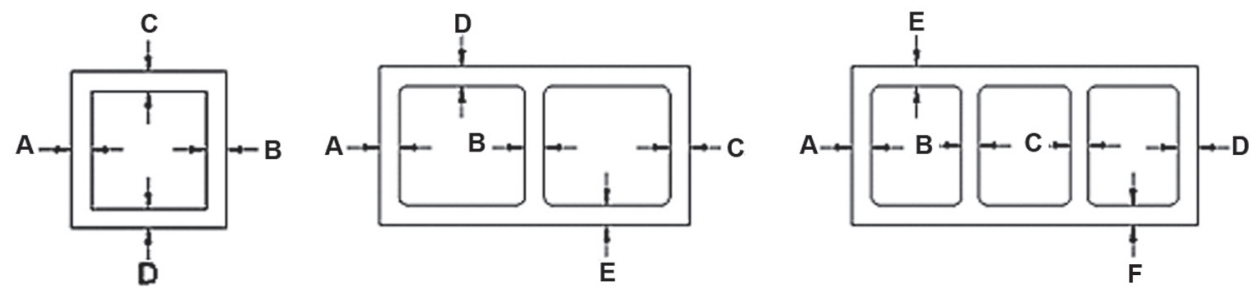

Figura 4. Medición de los espesores de pared de los bloques de 1, 2 y 3 celdas.

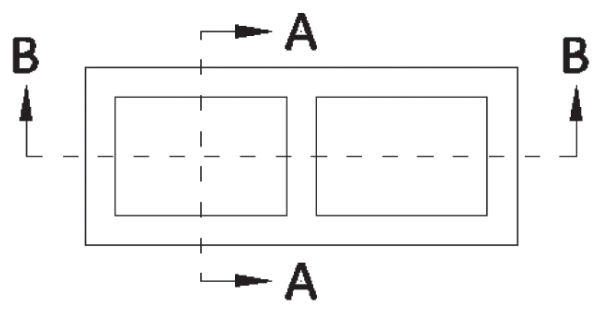

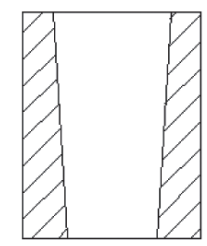

Sección A-A

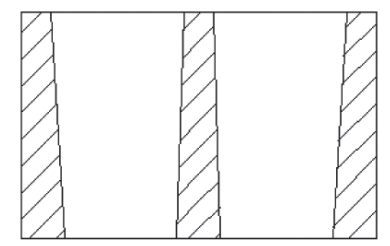

Sección B-B

Figura 5. Sección transversal de un bloque de concreto.

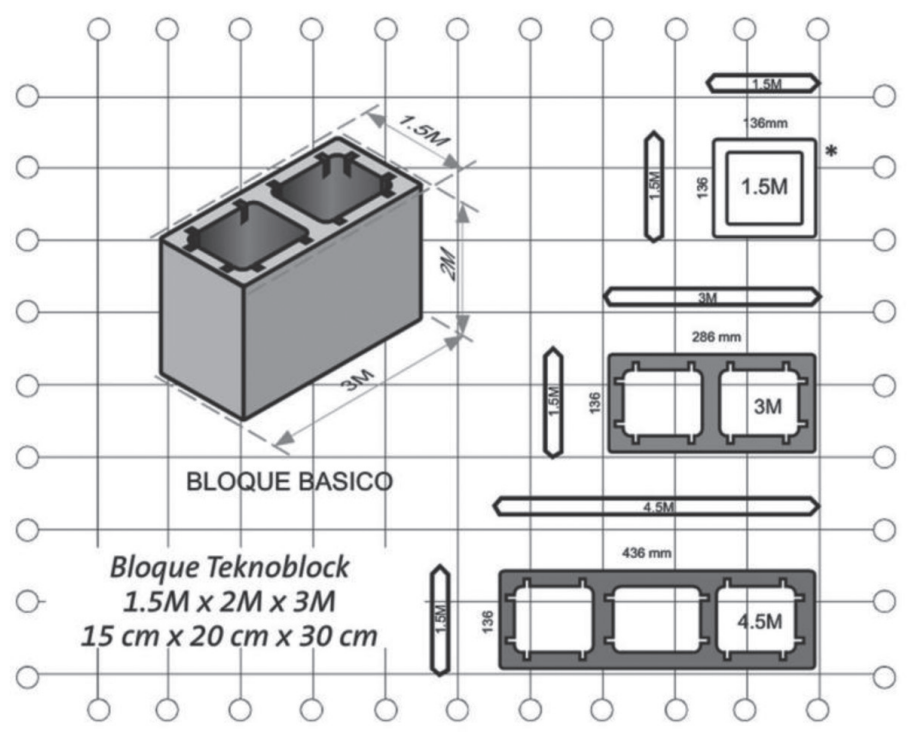

Figura 6. Bloques modulares tipo "Tecnoblock".

Nota: "Manual Técnico PC - Bloques de mampostería" por Productos de Concreto S.A, 2013. 
Tabla 4. Dimensiones reales promedio de los bloques estándar, según bloquera.

\begin{tabular}{lccccccccc}
\hline & \multicolumn{3}{c}{ Bloque 12 cm } & \multicolumn{3}{c}{ Bloque 15 cm } & \multicolumn{3}{c}{ Bloque 20 cm } \\
& $\begin{array}{c}\text { Espesor } \\
(\mathbf{c m})\end{array}$ & $\begin{array}{c}\text { Alto } \\
(\mathbf{c m})\end{array}$ & $\begin{array}{c}\text { Largo } \\
(\mathbf{c m})\end{array}$ & $\begin{array}{c}\text { Espesor } \\
(\mathbf{c m})\end{array}$ & $\begin{array}{c}\text { Alto } \\
(\mathbf{c m})\end{array}$ & $\begin{array}{c}\text { Largo } \\
(\mathbf{c m})\end{array}$ & $\begin{array}{c}\text { Espesor } \\
(\mathbf{c m})\end{array}$ & $\begin{array}{c}\text { Alto } \\
(\mathbf{c m})\end{array}$ & $\begin{array}{c}\text { Largo } \\
(\mathbf{c m})\end{array}$ \\
\hline Bloquera I & 12.1 & 19.2 & 39.0 & 15.0 & 19.2 & 39.0 & 20.0 & 18.9 & 38.9 \\
Bloquera II & 12.0 & 18.9 & 38.9 & - & - & - & 20.0 & 19.1 & 39.0 \\
Bloquera III & 12.1 & 19.3 & 39.1 & 15.0 & 19.0 & 39.0 & 20.1 & 18.9 & 39.0 \\
Bloquera IV & 12.0 & 19.1 & 39.0 & 15.0 & 19.0 & 39.0 & 20.0 & 18.9 & 39.2 \\
Bloquera V & 12.0 & 19.0 & 39.0 & 15.0 & 19.0 & 39.0 & 20.1 & 19.1 & 39.1 \\
Bloquera VI & 12.0 & 19.0 & 39.3 & 15.0 & 18.8 & 39.1 & 20.0 & 18.7 & 39.0 \\
Bloquera VII & 12.0 & 19.1 & 39.0 & 15.0 & 19.0 & 38.9 & 20.0 & 19.0 & 39.0 \\
Bloquera VIII & 12.0 & 19.0 & 39.1 & 15.0 & 19.0 & 39.1 & 20.0 & 19.1 & 39.0 \\
Bloquera IX & 12.0 & 18.9 & 39.0 & 15.0 & 19.0 & 38.9 & 20.0 & 19.0 & 39.0 \\
Bloquera X & 12.0 & 18.9 & 38.8 & 15.0 & 19.2 & 38.9 & 20.0 & 19.1 & 38.8 \\
Bloquera XI & 12.0 & 19.0 & 38.9 & 15.0 & 19.1 & 38.9 & 20.0 & 19.1 & 38.9 \\
Bloquera XII & 12.0 & 19.1 & 38.9 & 15.0 & 18.9 & 38.9 & 20.1 & 18.8 & 39.0 \\
Bloquera XIII & 12.0 & 18.9 & 38.9 & 15.0 & 18.9 & 39.0 & 20.0 & 19.0 & 38.8 \\
Bloquera XIV & 12.0 & 19.0 & 39.1 & 15.0 & 19.0 & 39.0 & 20.0 & 19.0 & 39.0 \\
Bloquera XV & 12.0 & 18.9 & 38.9 & 15.0 & 19.0 & 39.1 & 20.0 & 19.0 & 38.9 \\
Bloquera XVI & 12.0 & 18.8 & 39.0 & 15.1 & 18.9 & 39.2 & 20.1 & 18.9 & 39.1 \\
\hline & & & & & & & & & \\
& & & & & & & & &
\end{tabular}

Tabla 5. Dimensiones reales promedio de los bloques modulares, según bloquera.

\begin{tabular}{lccccccccccc}
\hline & \multicolumn{3}{c}{ Modular 15 cm } & \multicolumn{3}{c}{ Modular 30 cm } & \multicolumn{3}{c}{ Modular 45 cm } \\
& $\begin{array}{c}\text { Espesor } \\
(\mathbf{c m})\end{array}$ & $\begin{array}{c}\text { Alto } \\
(\mathbf{c m})\end{array}$ & $\begin{array}{c}\text { Largo } \\
(\mathbf{c m})\end{array}$ & $\begin{array}{c}\text { Espesor } \\
(\mathbf{c m})\end{array}$ & $\begin{array}{c}\text { Alto } \\
(\mathbf{c m})\end{array}$ & $\begin{array}{c}\text { Largo } \\
(\mathbf{c m})\end{array}$ & $\begin{array}{c}\text { Espesor } \\
(\mathbf{c m})\end{array}$ & $\begin{array}{c}\text { Alto } \\
(\mathbf{c m})\end{array}$ & $\begin{array}{c}\text { Largo } \\
(\mathbf{c m})\end{array}$ \\
\hline Bloquera I & 13.7 & 19.0 & 13.7 & 13.5 & 19.2 & 28.5 & 13.5 & 19.5 & 43.5 \\
Bloquera II & - & - & - & 13.6 & 18.4 & 28.6 & 13.6 & 18.5 & 43.6 \\
Bloquera III & - & - & - & - & - & - & 13.5 & 19.8 & 43.7 \\
Bloquera IV & - & - & - & 13.6 & 19.1 & 28.5 & - & - & - \\
\hline
\end{tabular}


Tabla 6. Dimensiones reales promedio de los bloques modulares, según bloquera.

\begin{tabular}{lccccccccccc}
\hline & \multicolumn{3}{c}{ Bloque 12 cm } & \multicolumn{3}{c}{ Bloque 15 cm } & \multicolumn{3}{c}{ Bloque $20 \mathrm{~cm}$} \\
& $\begin{array}{c}\text { Espesor } \\
(\mathbf{c m})\end{array}$ & $\begin{array}{c}\text { Alto } \\
(\mathbf{c m})\end{array}$ & $\begin{array}{c}\text { Largo } \\
(\mathbf{c m})\end{array}$ & $\begin{array}{c}\text { Espesor } \\
(\mathbf{c m})\end{array}$ & $\begin{array}{c}\text { Alto } \\
(\mathbf{c m})\end{array}$ & $\begin{array}{c}\text { Largo } \\
(\mathbf{c m})\end{array}$ & $\begin{array}{c}\text { Espesor } \\
(\mathbf{c m})\end{array}$ & $\begin{array}{c}\text { Alto } \\
(\mathbf{c m})\end{array}$ & $\begin{array}{c}\text { Largo } \\
(\mathbf{c m})\end{array}$ \\
\hline$\overline{\mathrm{x}}(\mathrm{cm})$ & 12.0 & 19.0 & 39.0 & 15.0 & 19.0 & 39.0 & 20.0 & 19.0 & 39.0 \\
$\mathrm{~s}(\mathrm{~cm})$ & 0.03 & 0.13 & 0.12 & 0.02 & 0.13 & 0.08 & 0.03 & 0.12 & 0.09 \\
\hline
\end{tabular}

Tabla 7. Promedio y desviación estándar de las dimensiones reales de los bloques modulares, según longitud.

\begin{tabular}{lccccccccccc}
\hline & \multicolumn{3}{c}{ Modular 15 cm } & \multicolumn{3}{c}{ Modular $30 \mathbf{c m}$} & \multicolumn{3}{c}{ Modular 45 cm } \\
& $\begin{array}{c}\text { Espesor } \\
(\mathbf{c m})\end{array}$ & $\begin{array}{c}\text { Alto } \\
(\mathbf{c m})\end{array}$ & $\begin{array}{c}\text { Largo } \\
(\mathbf{c m})\end{array}$ & $\begin{array}{c}\text { Espesor } \\
(\mathbf{c m})\end{array}$ & $\begin{array}{c}\text { Alto } \\
(\mathbf{c m})\end{array}$ & $\begin{array}{c}\text { Largo } \\
(\mathbf{c m})\end{array}$ & $\begin{array}{c}\text { Espesor } \\
(\mathbf{c m})\end{array}$ & $\begin{array}{c}\text { Alto } \\
(\mathbf{c m})\end{array}$ & $\begin{array}{c}\text { Largo } \\
(\mathbf{c m})\end{array}$ \\
\hline$\overline{\mathrm{x}}(\mathrm{cm})$ & 13.7 & 19.0 & 13.7 & 13.6 & 18.9 & 28.5 & 13.5 & 19.2 & 43.6 \\
$\mathrm{~s}(\mathrm{~cm})$ & - & - & - & 0.04 & 0.42 & 0.08 & 0.05 & 0.65 & 0.09 \\
\hline
\end{tabular}

modulares se refieren al Tecnoblock del ITCR, el cual se muestra en la Figura 6.

\section{PRESENTACIÓN Y ANÁLISIS DE RESULTADOS}

\subsection{Dimensiones reales del bloque de concreto}

En la Tabla 4 y 5 , se presentan los resultados de dimensiones promedio obtenidas en cada uno de los bloques muestreados en las bloqueras estudiadas, tanto de los bloques estándar como de los bloques modulares.

Al analizar la totalidad de la muestra, es decir, todas las dimensiones realizadas en cada uno de los bloques de cada tipo y de cada bloquera, se obtienen los resultados estadísticos (Ver Tablas 6 y 7 )

Con los resultados anteriores, se comprueba que las dimensiones promedio de los bloques son de 12 x 19 x $39 \mathrm{~cm}, 15$ x 19 x $39 \mathrm{~cm}$ y 20 x 19 x 39 $\mathrm{cm}$, en el caso del bloque estándar de $12 \mathrm{~cm}, 15$ $\mathrm{cm}$ y $20 \mathrm{~cm}$ de espesor respectivamente; mientras que en el caso del bloque modular se tiene $13.7 \mathrm{x}$ 19 x $13.7 \mathrm{~cm}, 13.6$ x 18.9 x $28.5 \mathrm{~cm}$ y 13.5 x 19.2 x $43.6 \mathrm{~cm}$ para los bloques de $15 \mathrm{~cm}, 30 \mathrm{~cm}$ y 45 $\mathrm{cm}$ de longitud nominal respectivamente.

\subsection{Espesores de pared del bloque de concreto}

En la Tabla 8, se presentan los resultados de los espesores de pared promedio obtenidos en cada uno de los bloques muestreados en las bloqueras estudiadas, tanto de los bloques estándar como de los bloques modulares. En este artículo, únicamente se presentan los resultados de los espesores de pared mínimos medidos, denominados como A, B, C, D, E y F.

Como ejemplo, en el caso del bloque de concreto de dos celdas de $20 \mathrm{~cm}$ de espesor, el espesor de pared interno se refiere a los valores A, 
Tabla 8. Espesor de pared menor promedio de los bloques de concreto estándar, según bloquera

\begin{tabular}{|c|c|c|c|c|}
\hline & Bloque $12 \mathrm{~cm}$ & Bloque $15 \mathrm{~cm}$ & $\begin{array}{c}\text { Bloque } 20 \mathrm{~cm} \\
\text { (int) }\end{array}$ & $\begin{array}{c}\text { Bloque } 20 \mathrm{~cm} \\
\text { (ext) }\end{array}$ \\
\hline & \multicolumn{4}{|c|}{$\mathrm{mm}$} \\
\hline Bloquera I & 27.4 & 27.5 & 31.3 & 33.2 \\
\hline Bloquera II & 25.1 & - & 31.1 & 33.1 \\
\hline Bloquera III & 27.7 & 27.0 & 30.3 & 33.4 \\
\hline Bloquera IV & 25.8 & 26.9 & 31.7 & 33.7 \\
\hline Bloquera V & 25.8 & 28.1 & 32.1 & 34.7 \\
\hline Bloquera VI & 26.8 & 28.1 & 29.2 & 28.4 \\
\hline Bloquera VII & 26.0 & 26.9 & 31.6 & 33.7 \\
\hline Bloquera VIII & 27.5 & 26.9 & 27.5 & 27.9 \\
\hline Bloquera IX & 24.5 & 28.3 & 31.2 & 32.6 \\
\hline Bloquera X & 27.2 & 27.1 & 31.0 & 32.9 \\
\hline Bloquera XI & 24.8 & 27.1 & 30.3 & 33.5 \\
\hline Bloquera XII & 27.3 & 27.2 & 28.2 & 34.1 \\
\hline Bloquera XIII & 26.8 & 27.3 & 30.2 & 33.2 \\
\hline Bloquera XIV & 25.8 & 26.5 & 31.2 & 33.9 \\
\hline Bloquera XV & 25.6 & 27.2 & 32.5 & 35.1 \\
\hline Bloquera XVI & 27.7 & 31.2 & 29.2 & 33.2 \\
\hline
\end{tabular}

Tabla 9. Espesor de pared menor promedio de los bloques de concreto modulares, según bloquera.

\begin{tabular}{lccc}
\hline & Modular $15 \mathrm{~cm}$ & Modular $30 \mathrm{~cm}$ & Modular $45 \mathrm{~cm}$ \\
\cline { 2 - 4 } & 26.1 & $\mathrm{~mm}$ & \\
\hline Bloquera IV & - & 26.1 & 26.2 \\
Bloquera IX & - & 26.4 & 26.8 \\
Bloquera X & - & - & 26.7 \\
Bloquera XV & & 25.6 & - \\
\hline
\end{tabular}


Tabla 10. Resultados estadísticos de los espesores de pared menores de los bloques de concreto.

\begin{tabular}{|c|c|c|c|c|c|c|c|}
\hline & $\begin{array}{l}\text { Bloque } \\
12 \mathrm{~cm}\end{array}$ & $\begin{array}{l}\text { Bloque } \\
15 \mathrm{~cm}\end{array}$ & $\begin{array}{c}\text { Bloque } \\
20 \mathrm{~cm} \text { (int) }\end{array}$ & $\begin{array}{c}\text { Bloque } \\
20 \mathrm{~cm}(\text { ext) }\end{array}$ & $\begin{array}{c}\text { Modular } \\
15 \mathrm{~cm}\end{array}$ & $\begin{array}{l}\text { Modular } \\
30 \mathrm{~cm}\end{array}$ & $\begin{array}{c}\text { Modular } \\
45 \mathrm{~cm}\end{array}$ \\
\hline$\overline{\mathrm{x}}(\mathrm{mm})$ & 26.4 & 27.5 & 30.5 & 32.9 & 26.1 & 26.0 & 26.6 \\
\hline $\mathrm{s}(\mathrm{mm})$ & 1.06 & 1.18 & 1.43 & 1.94 & - & 0.38 & 0.36 \\
\hline Mín. (mm) & 24.1 & 24.5 & 27.3 & 27.6 & 26.0 & 25.3 & 26.0 \\
\hline P5 (mm) & 24.6 & 26.7 & 27.6 & 28.1 & 26.0 & 25.4 & 26.1 \\
\hline
\end{tabular}

Tabla 11. Espesor de pared recomendado para los bloques de concreto estándar y modular.

\begin{tabular}{lccccc}
\hline & $\begin{array}{c}\text { Bloque } \\
\mathbf{1 2} \mathbf{~ c m}\end{array}$ & $\begin{array}{c}\text { Bloque } \\
\mathbf{1 5} \mathbf{~ c m}\end{array}$ & $\begin{array}{c}\text { Bloque } \\
\mathbf{2 0} \mathbf{~ c m}(\mathbf{i n t})\end{array}$ & $\begin{array}{c}\text { Bloque } \\
\mathbf{2 0} \mathbf{~ c m}(\mathbf{e x t})\end{array}$ & Modular \\
\hline tf - Norma INTE (mm) & 19 & 25 & 25 & 32 & 22.2 \\
tf - Recomendado (mm) & 24 & 26 & 28 & 2 & 25 \\
\% Aumento & $26 \%$ & $4 \%$ & $12 \%$ & $0 \%$ & $13 \%$ \\
\hline
\end{tabular}

B y C, mientras que el espesor externo se refiere a los valores D y E mostrados en la Figura 4.

Al analizar la totalidad de la muestra, es decir, todas las dimensiones realizadas en cada uno de los bloques de cada tipo y de cada bloquera, se obtienen los siguientes resultados estadísticos.

El percentil 5 (P5) calculado para cada espesor de pared de cada tipo de bloque, se utilizó con el fin de recomendar un espesor de pared mínimo para emplear en el diseño de la mampostería de cada tipo de bloque.

Los espesores de pared mínimos encontrados superan los espesores de pared mínimos establecidos por la norma INTE 06-03-01 (Tabla 3), con la excepción de 2 bloqueras que incumplen el espesor mínimo de pared externo del bloque de $20 \mathrm{~cm}$, según se aprecia en el Tabla
8. Además, se encontraron diferencias de entre 4 $\mathrm{mm}$ y $7 \mathrm{~mm}$ entre el espesor de pared menor y mayor del bloque de concreto.

En el Tabla 11 se aprecia el considerable aumento en el espesor de pared del bloque de concreto, especialmente en el bloque de $12 \mathrm{~cm}$ de espesor. En el caso del bloque de $20 \mathrm{~cm}$, dado que 2 bloqueras no cumplieron con el mínimo establecido, se recomienda el mismo valor establecido por la norma INTE 06-03-01 de 32 mm para el espesor de pared externo.

Cabe destacar que en el caso del bloque de $12 \mathrm{~cm}, 15 \mathrm{~cm}$ y modular, el aumento del espesor de pared significa un aumento de un 54\%, $8 \%$ y $26 \%$, respectivamente, en la capacidad en cortante de un muro de mampostería parcialmente relleno con cargas paralelas a su plano, según la ecuación 
[9-24] del CSCR10, la cual se basa en la ecuación [3-22] del TMS 402-08. En el caso del bloque de $20 \mathrm{~cm}$ de espesor, no se da este aumento en la capacidad debido a que el espesor de pared externo se mantiene igual al mínimo de la norma.

\subsection{Propiedades geométricas de la mampostería de concreto}

El ensayo de área neta incluyó un total de 162 bloques de concreto de los 270 muestreados para la medición de propiedades geométricas, de manera que se muestrearon 3 bloques de cada tipo en cada una de las bloqueras. Se utilizó este número de muestras pues se siguieron las indicaciones de la norma ASTM C140.

Las principales propiedades geométricas analizadas fueron:

- Área neta

- Espesor equivalente

- Inercia transversal neta

- Módulo de sección transversal neto

Según Armhein (1998), el espesor equivalente de la mampostería se define como el espesor que tendría el muro de mampostería si no existieran celdas vacías. Este valor depende del patrón de refuerzo vertical y del espesor del bloque de concreto.

Además, para calcular las propiedades geométricas, se emplearon los siguientes supuestos.

- $\quad$ Espesores de pared recomendados (Tabla 11)

- Sección de mampostería (Figura 7)

- Promedio de celdas vacías por metro (Figura 8)

- Análisis estadístico

- Percentil 5

En el caso del bloque modular, se analizaron 3 posibles configuraciones para construir una pared de $103.6 \mathrm{~cm}$ de longitud. Estas configuraciones se muestran en la Figura 9.

Para el cálculo del área neta de la mampostería, se utilizó el promedio de celdas vacías por metro según el patrón de refuerzo vertical y el promedio del área neta del bloque

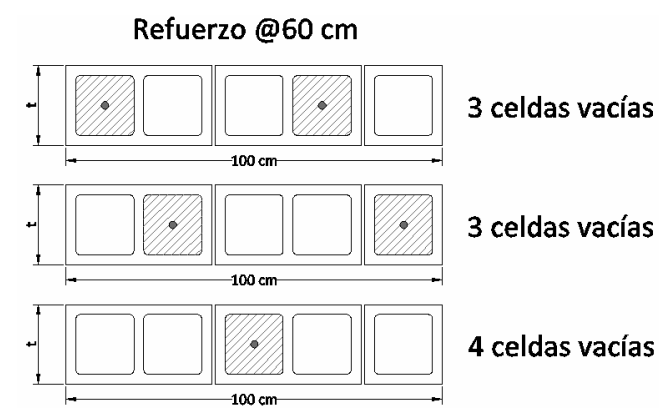

Figura 7. Cantidad de celdas vacías por metro de pared con el refuerzo a cada $60 \mathrm{~cm}$.

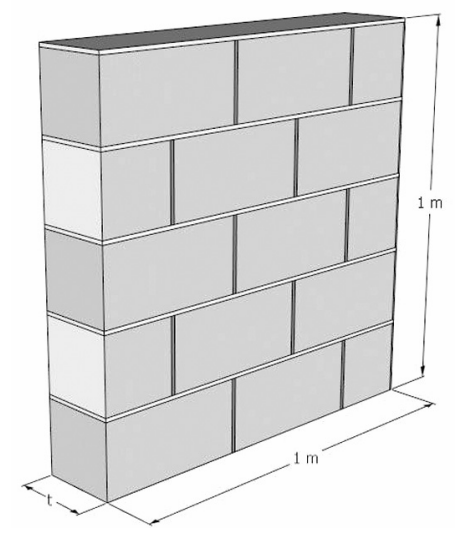

Figura 8. Pared de bloque de concreto de $1 \mathrm{~m} 2$ utilizada para el cálculo del espesor equivalente del bloque estándar.

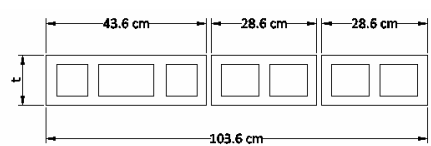

Opción 1
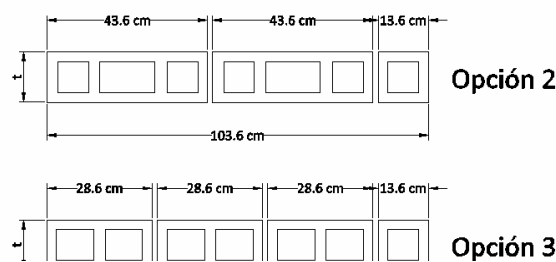

Figura 9. Opciones constructivas analizadas para una pared con bloque modular 
de concreto; con este valor y el volumen de la pared, se obtuvo el espesor equivalente de la mampostería. La inercia y módulo de sección transversal neta de la mampostería, en el eje débil, se obtuvieron al analizar una sección equivalente de mampostería con una única celda vacía, tomando en consideración su patrón de refuerzo vertical (Figura 10).

A continuación, se muestran las propiedades geométricas obtenidas para la mampostería con los diferentes tipos de bloque analizado.

Tabla 12. Propiedades geométricas de la mampostería con bloque estándar de $12 \mathrm{~cm}$ de espesor.

\begin{tabular}{ccccc}
\hline $\begin{array}{l}\text { Separación } \\
\text { del refuerzo } \\
\text { vertical }(\mathbf{c m})\end{array}$ & $\begin{array}{c}\text { An } \\
(\mathbf{c m} \mathbf{2} / \mathbf{m})\end{array}$ & $\begin{array}{c}\mathbf{I n}, \mathbf{x}-\mathbf{x} \\
(\mathbf{c m} \mathbf{4} / \mathbf{m})\end{array}$ & $\begin{array}{c}\text { Sn, } \mathbf{x}-\mathbf{x} \\
(\mathbf{c m} \mathbf{3} / \mathbf{m})\end{array}$ & $\begin{array}{c}\text { tfe } \\
(\mathbf{c m})\end{array}$ \\
\hline 20 & 1200 & 14400 & 2400 & 12 \\
40 & 928 & 13266 & 2211 & 9.3 \\
60 & 837 & 12888 & 2148 & 8.4 \\
80 & 792 & 12699 & 2116 & 7.9 \\
\hline
\end{tabular}

Tabla 14. Propiedades geométricas de la mampostería con bloque estándar de $20 \mathrm{~cm}$ de espesor.

\begin{tabular}{ccccc}
\hline $\begin{array}{c}\text { Separación } \\
\text { del refuerzo } \\
\text { vertical }(\mathbf{c m})\end{array}$ & $\begin{array}{c}\text { An } \\
(\mathbf{c m} 2 / \mathbf{m})\end{array}$ & $\begin{array}{c}\mathbf{I n}, \mathbf{x}-\mathbf{x} \\
(\mathbf{c m} 4 / \mathbf{m})\end{array}$ & $\begin{array}{c}\text { Sn, } \mathbf{x}-\mathbf{x} \\
(\mathbf{c m} 3 / \mathbf{m})\end{array}$ & $\begin{array}{c}\text { tfe } \\
(\mathbf{c m})\end{array}$ \\
\hline 20 & 2000 & 66667 & 6667 & 20 \\
40 & 1483 & 58691 & 5869 & 14.8 \\
60 & 1310 & 56032 & 5603 & 13.1 \\
80 & 1224 & 54703 & 5470 & 12.2 \\
\hline
\end{tabular}

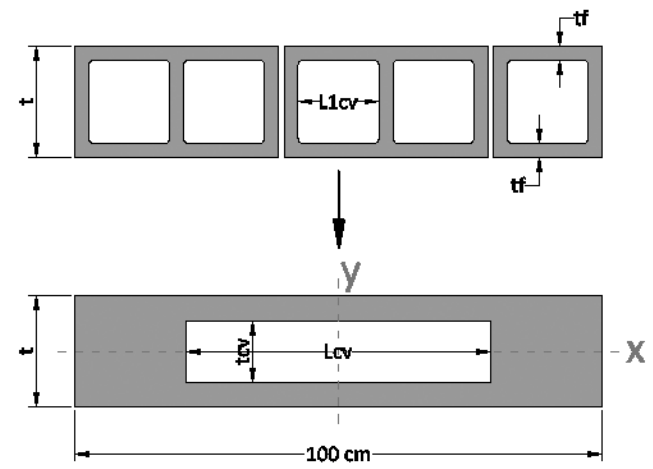

Figura 10. Sección equivalente de mampostería utilizada para el cálculo de las propiedades geométricas.

Tabla 13. Propiedades geométricas de la mampostería con bloque estándar de $15 \mathrm{~cm}$ de espesor.

\begin{tabular}{ccccc}
\hline $\begin{array}{l}\text { Separación } \\
\text { del refuerzo } \\
\text { vertical }(\mathbf{c m})\end{array}$ & $\begin{array}{c}\text { An } \\
(\mathbf{c m} \mathbf{2} / \mathbf{m})\end{array}$ & $\begin{array}{c}\mathbf{I n}, \mathbf{x}-\mathbf{x} \\
(\mathbf{c m} \mathbf{4} / \mathbf{m})\end{array}$ & $\begin{array}{c}\mathbf{S n}, \mathbf{x}-\mathbf{x} \\
(\mathbf{c m} \mathbf{3} / \mathbf{m})\end{array}$ & $\begin{array}{c}\text { tfe } \\
(\mathbf{c m})\end{array}$ \\
\hline 20 & 1500 & 28125 & 3750 & 15 \\
40 & 1142 & 25333 & 3378 & 11.4 \\
60 & 1022 & 24402 & 3254 & 10.2 \\
80 & 962 & 23937 & 3192 & 9.6 \\
\hline
\end{tabular}

Tabla 15. Propiedades geométricas de la mampostería con bloque modular (1).

\begin{tabular}{ccccc}
$\begin{array}{c}\text { Separación } \\
\text { del refuerzo } \\
\text { vertical }(\mathbf{c m})\end{array}$ & $\begin{array}{c}\text { An } \\
(\mathbf{c m} 2 / \mathbf{m})\end{array}$ & $\begin{array}{c}\mathbf{I n}, \mathbf{x}-\mathbf{x} \\
(\mathbf{c m} 4 / \mathbf{m})\end{array}$ & $\begin{array}{c}\text { Sn, } \mathbf{x}-\mathbf{x} \\
(\mathbf{c m} 3 / \mathbf{m})\end{array}$ & $\begin{array}{c}\text { tfe } \\
(\mathbf{c m})\end{array}$ \\
\hline 20 & 1409 & 21717 & 3194 & 13.6 \\
40 & 1102 & 19858 & 2920 & 10.7 \\
60 & 1014 & 19327 & 2842 & 9.8 \\
80 & 948 & 18929 & 2784 & 9.2 \\
\hline
\end{tabular}

Nota: (1) El bloque modular se refiere al Tecknoblock desarrollado por el ITCR, ver Figura 6. 
Para el cálculo de las propiedades geométricas, se emplearon los valores de percentil 5 del espesor de pared menor y área neta, y no los valores mínimos establecidos por la norma INTE. Por lo tanto, se generó un espesor y longitud de la celda vacía equivalente menor y por ende, mayor inercia, módulo de sección y propiedades geométricas en general, en todas las secciones analizadas con los diferentes tipos de bloque.

En el caso del bloque modular, se observó que las 3 opciones analizadas presentan resultados bastante similares entre ellos en todas las propiedades geométricas, de manera que se presentan los resultados más conservadores en la Tabla 15 y que corresponden a la opción 1, la cual se compone de los bloques con mayor área de una celda vacía, tanto individualmente como en la sección en general. En general, las propiedades geométricas de la mampostería disminuyen conforme se aumenta la separación del refuerzo vertical en todos los tipos de bloques, debido al aumento del promedio de celdas vacías por metro, por lo que se obtiene una pared equivalente con una sección hueca mayor.

\section{CONCLUSIONES Y RECOMENDACIONES}

\subsection{Bloques de concreto en Costa Rica}

1. El $35 \%$ de las bloqueras de mayor producción en Costa Rica se encuentran distribuidas entre las provincias de Alajuela y San José, mientras que el $45 \%$ se encuentran distribuidas en la Gran Área Metropolitana.

2. El cantón de Limón no cuenta con ninguna bloquera, mientras que el Pacífico Sur únicamente cuenta con una de las bloqueras de mayor producción en el país.

3. Todas las bloqueras de mayor producción fabrican bloque estándar de $12 \mathrm{~cm}, 15 \mathrm{~cm}$ y $20 \mathrm{~cm}$ de espesor; mientras que únicamente 4 bloqueras fabrican bloque modular.

4. El $80 \%$ de las bloqueras utiliza material de río como agregado para fabricar los bloques de concreto, mientras que el restante $20 \%$ utiliza material de tajo.

\subsection{Propiedades geométricas del bloque y la mampostería de concreto}

5. Las dimensiones promedio de los bloques de concreto estándar son de 12x19x39 cm, $15 \times 19 \times 39 \mathrm{~cm}$ y $20 \times 19 \times 39 \mathrm{~cm}$.

6. Las dimensiones promedio de los bloques de concreto modular son de $13.7 \times 19 \times 13.7 \mathrm{~cm}$, $13.6 \times 18.9 \times 28.5 \mathrm{~cm}$ y $13.5 \times 19.2 \times 43.6 \mathrm{~cm}$.

7. El percentil 5 del espesor de pared de todos los bloques de concreto estudiados, excepto el espesor de pared externo del bloque estándar de $20 \mathrm{~cm}$ de espesor, es superior al mínimo establecido por la norma INTE 06-03-01 para cada tipo de bloque.

8. El $12 \%$ de las bloqueras estudiadas incumplen el espesor de pared externo para el bloque estándar de $20 \mathrm{~cm}$ de espesor.

9. La relación promedio entre el área neta y el área bruta de los bloques de concreto es menor de 0.75 en todos los tipos de bloque analizados, por lo que clasifican como mampostería hueca según la ASTM C90.

10. La inercia y módulo de sección transversales netos de la mampostería son mayores a lo esperado, esto como resultado del mayor espesor de pared encontrado en todos los bloques de concreto, con respecto al espesor de pared mínimo establecido por la norma INTE 06-03-01.

11. Los resultados más conservadores de la mampostería modular se obtuvieron al considerar una sección de pared con bloques de $30 \mathrm{~cm}$ y $45 \mathrm{~cm}$ de longitud nominal.

12. Se recomienda que este tipo de estudios se lleven a cabo en el tiempo, dado que las condiciones de producción pueden variar, por ejemplo a causa del implemento de moldes nuevos, su ajuste, reparación y desgaste, o por la introducción de nuevos procedimientos de fabricación. Sin embargo, se considera que estas variaciones no ocurren de manera frecuente.

\section{SIMBOLOGÍA}

$\bar{x} \quad$ Promedio de la muestra, según la variable en análisis 
$\mathrm{A}_{\mathrm{n}}$ Área transversal neta del bloque de concreto

ASTM American Society for Testing and Materials

Bloque $12 \mathrm{~cm} \quad$ Bloque de concreto estándar de $12 \mathrm{~cm}$ de espesor

Bloque $15 \mathrm{~cm} \quad$ Bloque de concreto estándar de $15 \mathrm{~cm}$ de espesor

Bloque $20 \mathrm{~cm}$ (ext) Bloque de concreto estándar de $20 \mathrm{~cm}$ de espesor, espesor de pared externo

Bloque $20 \mathrm{~cm}$ (int) Bloque de concreto estándar de $20 \mathrm{~cm}$ de espesor, espesor de pared interno

Bloque $20 \mathrm{~cm} \quad$ Bloque de concreto estándar de $20 \mathrm{~cm}$ de espesor

CSCR Código Sísmico de Costa Rica

CSCR10 Código Sísmico de Costa Rica 2010

H Altura del bloque de concreto

\section{IBC International Building Code}

$I_{n, x-x} \quad$ Inercia transversal neta del bloque de concreto en el eje " $x$ " (eje débil)

INTECO Instituto de Normas Técnicas de Costa Rica

ITCR Instituto Tecnológico de Costa Rica

L Longitud del bloque de concreto

$\mathrm{L}_{\text {lcv }}$ Longitud de 1 celda vacía del bloque de concreto

Lanamme UCR Laboratorio Nacional de Materiales y Modelos Estructurales

$\mathrm{L}_{\mathrm{cv}} \quad$ Longitud de la celda vacía equivalente

MEIC Ministerio de Economía, Industria y Comercio
Modular $15 \mathrm{~cm}$ Bloque de concreto modular de $15 \mathrm{~cm}$ de longitud nominal

Modular $30 \mathrm{~cm}$ Bloque de concreto modular de $30 \mathrm{~cm}$ de longitud nominal

Modular $45 \mathrm{~cm}$ Bloque de concreto modular de $45 \mathrm{~cm}$ de longitud nominal

NCMA National Concrete Masonry Association

$\mathrm{P}_{5}$ Percentil 5 de la muestra, según la variable en análisis

s Desviación estándar de la muestra, según la variable en análisis

$\mathrm{S}_{\mathrm{n}, \mathrm{x}-\mathrm{x}}$ Módulo de sección transversal neto del bloque de concreto en el eje " $x$ " (eje débil)

t Espesor del bloque de concreto

$\mathrm{t}_{\mathrm{cv}} \quad$ Espesor de la celda vacía equivalente

Tecknoblock Bloques de concreto modulares desarrollados como parte de un estudio del ITCR y considerados como bloques modulares en este trabajo

tf Espesor de pared del bloque de concreto

$\mathrm{t}_{\mathrm{fe}}$ Espesor de pared equivalente de la mampostería

TMS 402-08 Building code requirements and specifications for masonry structures 2008

Viga bloque Bloque de concreto con una ranura, la cual se utiliza para colocar el refuerzo horizontal y que el mismo quede completamente embebido en concreto.

\section{REFERENCIAS BIBLIOGRÁFICAS}

American Standard for Testing Materials (ASTM). (2011).C140: Standard testmethod for sampling and testing concrete masonry units and related units. Pennsylvania: ASTM International. 
American Standard for Testing Materials (ASTM).(2011).C90: Standard specification for loadbearing concrete masonry units. Pennsylvania: ASTM International.

Armhein, J. E. (1998). Reinforced Masonry Engineering Handbook, clay and concrete masonry (5a ed). Florida: Masonry Institute of America.

Colegio Federado de Ingenieros y Arquitectos (CFIA). (2011). Código Sísmico de Costa Rica 2010. San José: Editorial Tecnológica de Costa Rica.

Instituto de Normas Técnicas de Costa Rica (INTECO).(2007).INTE06-02-13:Muestreo y ensayo de unidades de mampostería de concreto (bloques de concreto). San José: Editorial Tecnológica de Costa Rica.

Instituto de Normas Técnicas de Costa Rica (INTECO). (2007). INTE 06-03-01: Elementos de mampostería hueca de concreto (bloques de concreto) para uso estructural - Requisitos. San José: Editorial Tecnológica de Costa Rica.

International Code Council.(2003). International Building Code Commentary. Washington: International Code Council.

Masonry Standards Joint Committee (MSJC). (2008). Building Code Requirements and
Specification for Masonry Structures. Virginia: The Masonry Society.

National Concrete Masonry Association (NCMA). (2007). TEK Manual 14-1B: Section properties of concrete masonry walls. Virginia: NCMA.

National Concrete Masonry Association (NCMA). (2012). TEK Manual 18-2b: Sampling and testing concrete masonry units. Virginia: NCMA.

Productos de Concreto S.A. (2013) Manual Técnico PC - Bloques de mampostería. Recuperado de http://pc.cr/catalogo/ catalogo_mamposteria/files/assets/basichtml/page6.html

\section{SOBRE LOS AUTORES}

Alejandro Navas Carro: Universidad de Costa Rica. Magister en Ingeniería Civil. Profesor de la Escuela de Ingeniería Civil; Director del Laboratorio Nacional de Materiales y Modelos Estructurales, Universidad de Costa Rica. ALEJANDRO.NAVAS@ucr.ac.cr

Julio César Salazar Méndez: Empresa Constructora Proycon, Asistente de Ingeniero de Proyecto; Licenciado en Ingeniería Civil Universidad de Costa Rica. juliocesarcr@ gmail.com 